\title{
Bevacizumab in the treatment of HER2-negative breast cancer
}

\author{
Vito Lorusso \\ Oncologic Institute, Vito Fazzi \\ Hospital, Lecce, Italy
}

Correspondence: Vito Lorusso P.zza Muratore, I-73I00 Lecce, Italy

Tel/Fax +390832 661962

Email vitolorusso@inwind.it

\begin{abstract}
Angiogenesis has a clear and definite role in the breast cancer progression process, making antivascular endothelial growth factor (VEGF) therapies an attractive option for the treatment of metastatic breast cancer (MBC). Bevacizumab is a potent humanized monoclonal antibody to VEGF, which has shown regression of breast cancer in preclinical and clinical setting, either alone or in combination with cytotoxic treatment. Additionally, bevacizumab potentially increases the effectiveness of other anticancer therapies through the normalization of tumor vasculature, reduction of intratumoral pressure and improved tumor oxygenation. Phase $1 / 2$ trials showed significant antitumor effects of bevacizumab in $\mathrm{MBC}$, in particular in tumors not expressing HER2 receptor. A first phase 3 trial in pre-treated MBC patients showed better response rates but no survival benefit from the addition of bevacizumab to capecitabine. However, in two phase 2 trial in first-line setting in patients with MBC, bevacizumab improved progression-free survival in combination with weekly paclitaxel in comparison to paclitaxel alone or in combination with 3-weekly docetaxel in comparison with docetaxel alone, respectively. Bevacizumab in combination with taxanes seems to be a highly effective first-line treatment for MBC patients. Future research will investigate bevacizumab in the neoadjuvant or adjuvant setting, where even more potential may exist for these patients.
\end{abstract}

Keywords: bevacizumab, breast cancer, HER2, HER2-negative breast cancer

\section{Introduction}

Breast cancer represents a heterogeneous array of different disease subtypes with unique molecular phenotypes and distinct clinical features (Slamon et al 2006). Despite advances in the treatment of early-stage breast cancer, approximately one third of patients will eventually develop metastatic breast cancer (MBC) (Early Breast Cancer Trialists' Collaborative Group 1998). The prognosis of patients with MBC is poor, with a median survival time ranging from 24 to 48 months (Gennari et al 2005). Recently, advances in understanding the biology of breast cancer have led to the classification of breast tumors based upon their molecular features and the advent of targeted therapies for the treatment of both early and MBC. Targeted agents and their promise of better patient outcome with respect to safety, survival, and quality of life may change the clinical course for many MBC patients. Vascular endothelial growth factor (VEGF) is a critical mediator in tumor angiogenesis for many solid malignancies, including breast cancer (Ferrara et al 1997). Upon binding to its receptor, VEGF induces a cascade of intracellular signals inducing cellular proliferation, increased vaso-permeability, inhibition of apoptosis, and ultimately angiogenesis. For many tumors, VEGF appears to be a rate-limiting signal in angiogenesis, making it an attractive target for therapeutic agents. Bevacizumab is a humanized recombinant antibody that prevents VEGF receptor binding, and inhibits angiogenesis and tumor growth (Manley et al 2002). Its use has been recently approved in the US and in the 
EU for colorectal cancer, breast cancer, non-small cell lung cancer, and renal cell carcinoma (Genentech Inc., European Medicines Agency).

\section{Preclinical data}

Preclinical data suggested the potential for synergy by combining targeted antiangiogenic agents with traditional cytotoxic chemotherapy (Kakeji et al 1997; Gasparini et al 2005). Anti-VEGF therapies may help to "normalize" the chaotic architecture of vessels within tumors, reducing vascular permeability and interstitial fluid pressure, and potentially improving cytotoxic drug delivery (Jain 2005). In murine breast cancer models, twice-weekly intraperitoneal administration of A.4.6.1 (an antibody parent to bevacizumab), at a dose of $200 \mu \mathrm{g}$, significantly suppressed angiogenic activity (Borgstrom et al 1999). Doxorubicin alone also reduced the growth rate of MCF-7 cells but did not affect angiogenesis significantly. In contrast, doxorubicin with A.4.6.1 significantly reduced tumor regression, so that viable tumor cells could not be detected in some animals at the end of the 2-week observation period. In another trial, endothelial-cell stimulation with VEGF and bFGF protected endothelial cells from the antiangiogenic properties of docetaxel, but protection was lost when bevacizumab was co-administered with docetaxel, both in vitro and in vivo (Sweeney et al 2001). These data strongly support the view that neutralization of VEGF with bevacizumab, in combination with conventional cytotoxic agents, could be a promising treatment for $\mathrm{MBC}$.

\section{Phase I and early phase 2 studies}

Two phase 1 clinical trials of bevacizumab have been reported. In a phase 1 safety and pharmacokinetic study, bevacizumab was administered to 25 patients with refractory solid tumors at escalating doses ranging from 0.1 to $10 \mathrm{mg} / \mathrm{kg}$ (Gordon et al 2001) over 6 weeks. In a phase $1 \mathrm{~b}$ trial, bevacizumab, at a dose of $3 \mathrm{mg} / \mathrm{kg}$, was administered in combination with chemotherapy to 12 patients with advanced cancer (Margolin et al 2001). Those trials showed that bevacizumab can be administered safely, without dose-limiting toxicities, at doses up to $10 \mathrm{mg} / \mathrm{kg}$, and that it can be combined with chemotherapy without apparent synergistic toxicity. All patients who completed 6-12 months of therapy in the phase $1 / 2$ trials of bevacizumab were given the opportunity to participate in an ongoing extension study (Langmuir et al 2002). Of 52 patients with advanced solid tumors, 28 received bevacizumab for 1 year or more. The dosage of bevacizumab ranged from 5 to $15 \mathrm{mg} / \mathrm{kg}$ every 2 or 3 weeks. The majority of patients treated for 1 year had an observation period off therapy up to 6 months, but were able to restart bevacizumab at progression. Sixteen patients progressed on or before the observation period and restarted bevacizumab. The median duration of treatment was 14 months (range 11-36 months) and, at the time of reporting these results, a median survival time had not been reached (range 17 months to $>40$ months, with 20 patients alive).

In a phase $1 / 2$ dose-finding trial involving 75 women heavily pre-treated with $\mathrm{MBC}$, patients were assigned to receive single-agent bevacizumab in escalating doses ranging from $3 \mathrm{mg} / \mathrm{kg}$ to $20 \mathrm{mg} / \mathrm{kg}$ every other week (Cobleigh et al 2003). The majority of patients ( $83 \%$ ) had infiltrating ductal carcinoma, and $96 \%$ had received prior anthracycline- or taxane-based therapy for metastatic disease. An overall response rate (ORR) of $9.3 \%$ was reported, with $17 \%$ of patients maintaining stable disease or better after 5 months of therapy. Four patients continued therapy for at least 1 year without progression. Twenty-one of the 75 (28\%) patients were HER2 positive and $47(63 \%)$ were HER2 negative. Twelve of the 75 patients $(16 \%)$ completed the 6-month trial and received all 13 scheduled doses of bevacizumab. The median number of doses of bevacizumab administered was 6 and the median duration of treatment was 70 days. Treatment was well tolerated, with hypertension and proteinuria the most commonly reported toxicities. A phase 2 trial of bevacizumab with vinorelbine examined patients with MBC. Key eligibility criteria included prior chemotherapy with 1 or 2 regimens for $\mathrm{MBC}$ (including trastuzumab for HER2-positive disease) and disease progression within 1 year of adjuvant chemotherapy. Patients received treatment with bevacizumab at a dose of $10 \mathrm{mg} / \mathrm{kg}$ every 2 weeks and vinorelbine at a dose of $25 \mathrm{mg} / \mathrm{m}^{2} /$ week (adjusted for neutrophil count) until either the disease progresses or they experience undue toxicity. That trial has observed 17 responses (1 complete and 16 partial) among 55 patients (31\% [ORR]). Overall, bevacizumab with vinorelbine was well tolerated, and toxicity analyses indicate only minor occurrences of hypertension, proteinuria, and epistaxis and one instance of pericardial effusion. Side effects were consistent with the historic experience of the use of vinorelbine with neither bleeding nor thrombotic major events noted (Burstein et al 2002).

\section{Phase 3 trials}

These data supported the initiation of a phase 3 clinical trial that combined bevacizumab with capecitabine in patients previously treated with anthracyclines and/or taxanes. Miller and colleagues (Miller et al 2005) compared capecitabine with capecitabine plus bevacizumab (study AVF2119 g). 
This randomized phase 3 trial enrolled 462 women with $\mathrm{MBC}$ who had progressed following prior treatment with both an anthracycline- and taxane-containing regimen, and at least 1 but no more than 2 prior treatments for their MBC. Patients with HER-2 positive disease were permitted to be enrolled if they had had prior trastuzumab therapy. Patients were treated with capecitabine $2500 \mathrm{mg} / \mathrm{m}^{2}$ twice daily for days $1-14$ on a 21 -day cycle, alone or in combination with bevacizumab $15 \mathrm{mg} / \mathrm{kg}$ IV every 3 weeks.

Though response rate was significantly increased in patients treated with combined therapy $(19.8 \%$ vs $9.1 \%$; $\mathrm{p}=0.001)$, there was no difference in progression-free survival (PFS), the primary endpoint of the study. Combination therapy was well tolerated and appeared safe, with no apparent increase in capecitabine-related toxicities. Again, hypertension and proteinuria were the predominant toxicities, with no reported grade 4 events for either. The failure to achieve a statistically significant improvement in PFS may have been due to the refractory patient population and overall low response rates, as well as the failure to identify the cohort of patients for whom the targeted agents would be more effective. Indeed, angiogenic pathways become more numerously redundant as breast cancer progresses, suggesting that the optimal time for anti-VEGF therapies is earlier in the course of the disease (Relf et al 1997). This hypothesis led to a second phase 3 trial evaluating the incorporation of bevacizumab in the first-line chemotherapy for MBC.

The study E2100 (Miller et al 2007) randomized 722 women with locally recurrent or MBC (almost all HER2-negative) to receive either single-agentweekly paclitaxel $\left(90 \mathrm{mg} / \mathrm{m}^{2}\right.$ intravenously days 1,8 , and 15 every 4 weeks) or the same dose/schedule of paclitaxel plus bevacizumab (10 mg/kg days 1 and 15). Paclitaxel plus bevacizumab significantly prolonged progression-free survival as compared with paclitaxel alone (median, 11.8 vs 5.9 months; hazard ratio (HR) for progression, $0.60 ; \mathrm{p}<0.001$ ) and increased the objective response rate $(36.9 \%$ vs $21.2 \%, \mathrm{p}<0.001)$. Combined therapy increased the 1 -year survival rate $(81.2 \%$ vs $73.4 \%$, $\mathrm{p}=0.01$ ); however, the median overall survival was similar in the group receiving combined therapy and in the group receiving paclitaxel alone (26.7 months and 25.2 months, respectively; HR, $0.88 ; \mathrm{p}=0.16)$. Grade 3 or 4 hypertension $(14.8 \%$ vs $0.0 \%$, p $<0.001)$, proteinuria $(3.6 \%$ vs $0.0 \%$, $\mathrm{p}<0.001)$, headache $(2.2 \%$ vs $0.0 \%, \mathrm{p}=0.008)$, and cerebrovascular ischemia $(1.9 \%$ vs $0.0 \%, \mathrm{p}=0.02)$ were more frequent in patients receiving paclitaxel plus bevacizumab. Infection was more common in patients receiving paclitaxel plus bevacizumab $(9.3 \%$ vs $2.9 \%$, p $<0.001)$, but febrile neutropenia was uncommon ( $<1 \%$ overall). The authors concluded that initial therapy paclitaxel plus bevacizumab prolongs PFS, but not overall survival (OS), as compared with paclitaxel alone in MBC. However, this benefit in PFS a role for bevacizumab in the treatment of $\mathrm{MBC}$, and provides the impetus for further studies to investigate and refine the use of this potentially powerful agent. The XCALIBr trial evaluated capecitabine/bevacizumab in the first-line treatment of patients with MBC (Miller et al 2006). Median time to progression (TTP; $\mathrm{N}=106)$ was 5.7 months (95\% confidence interval [CI], 4.9-8.4 months); patients with estrogen receptor-positive disease $(n=57)$ had a superior outcome, with a median TTP of 8.9 months (95\% CI, 7.5-13.6 months), compared with 4 months (95\% CI, 3-4.9 months) in patients with estrogen receptor-negative disease. Furthermore, ORR was 38\% ( $47 \%$ in the estrogen receptor-positive population and $27 \%$ in the estrogen receptor-negative population), and median OS was $>16$ months $(>16.6$ months in patients with estrogen receptor-positive disease and $>7.5$ months in patients with estrogen receptor-negative disease). Capecitabine/ bevacizumab was well tolerated in this previously untreated population; the majority of AEs were mild or moderate. Thirteen percent of patients reported grade 3 palmar-plantar erythrodysesthesia, $10 \%$ reported grade 3 pain, and (in the first phase of the study) $2 \%$ reported grade 4 pulmonary embolism.

The benefit of adding bevacizumab to a taxane in the first-line treatment of $\mathrm{MBC}$ has been confirmed in another trial. Results from the AVADO study, reported at the American Society of Clinical Oncology 44th Annual Meeting (Miles et al 2008) showed a significant improvement in PFS, echoing the results of the E2100 study. AVADO used docetaxel and had a placebo group. Moreover, the trial also used different bevacizumab doses: E2100 used $10 \mathrm{mg} / \mathrm{kg}$; and AVADO compared a high dose of $15 \mathrm{mg} / \mathrm{kg}$ and a low dose of $7.5 \mathrm{mg} / \mathrm{kg}$. After a median follow-up of 11 months, the AVADO trial showed a statistically significant difference in PFS between women taking bevacizumab plus docetaxel and those taking docetaxel alone; stratified HR was 0.69 $(p=0.0035)$ for the low dose and $0.61(p=0.0001)$ for the high dose. In other words, the risk for disease progression was reduced by $21 \%$ with the low dose and by $29 \%$ with the high dose. The median TTP progression was 8 months with docetaxel alone, compared with 8.7 months with docetaxel plus low-dose bevacizumab, and 8.8 months with docetaxel plus high-dose bevacizumab. The ORR, a secondary end point, was $44 \%$ with docetaxel alone, $55 \%$ with low-dose bevacizumab ( $\mathrm{p}=0.0295)$, and $63 \%$ with high-dose bevacizumab 
$(p=0.0001)$. At the median follow-up of 11 months, $80 \%$ of the patients were still alive. Treatment-related deaths were $50(21 \%)$ after docetaxel alone, $49(20 \%)$ after low-dose bevacizumab, and 37 (15\%) after high-dose bevacizumab, giving a 1 -year survival of $73 \%, 78 \%$, and $83 \%$, respectively. A final analysis of OS data is expected by April 2009.

\section{Maximizing antiangiogenic activity of cytotoxic agents}

Several chemotherapeutic agents used routinely in cancer treatment are known to exert antiangiogenic activity. Maximal antiangiogenic activity typically requires prolonged exposure to low drug concentrations, which is in direct contrast to the strategy of using maximum tolerated doses when optimal tumor cell kill is the goal (Bocci et al 2002; Colleoni et al 2002; Kerbel et al 2004). Three reports (Kusaka et al 1994; Muramaki et al 2005; Higgins et al 2007) have confirmed the importance of dose and schedule in preclinical models. In all three, the combination of low, frequent-dose chemotherapy plus an agent that specifically targets the endothelial cell compartment (TNP-470 and anti-VEGF-2) controlled tumor growth much more effectively than the cytotoxic agent alone. These studies suggest that activated endothelial cells could be more sensitive, or even selectively sensitive, to protracted low-dose chemotherapy compared with other types of normal cells, thus creating a potential therapeutic window. Thus so far, only a few clinical trials have tested antiangiogenic schedules of chemotherapy - the so-called "metronomic therapy" (Emmenegger et al 2007). A phase 2 study (Colleoni et al 2002) of low-dose methotrexate (2.5 mg twice daily for 2 days each week) and cyclophosphamide (50 mg daily) in patients with previously treated MBC found an ORR rate of $19 \%$ (an additional 13\% of patients were stable for 6 months or more). Serum VEGF levels decreased in all patients remaining on therapy for at least 2 months but did not correlate with response. The preclinical data suggest that metronomic chemotherapy will be more effective when combined with a second antiangiogenic agent. To test this hypothesis, Burstein et al (2005) enrolled 55 patients with $\mathrm{MBC}$ and no more than 1 prior chemotherapy regimen in a randomized phase 2 trial of metronomic cyclophosphamide $(50 \mathrm{mg} /$ day $)$ and methotrexate $(2.5 \mathrm{mg}$ twice daily on days 1 and 2 each week) alone or with bevacizumab $(10 \mathrm{mg} / \mathrm{kg}$ every 2 weeks). ORR was $10 \%$ in the 21 patients treated with metronomic therapy alone and $29 \%$ in the 34 patients treated with metronomic chemotherapy plus bevacizumab. More recently, Dellapasqua et al reported the results of a phase 2 study with metronomic cyclophosphamide (50 mg daily) and capecitabine (500 mg twice daily) combined with bevacizumab $(10 \mathrm{mg} / \mathrm{kg}$ every 2 weeks) in MBC (Dellapasqua et al 2008).

In 46 assessable patients, they observed one complete response (CR; 2\%), 21 partial responses (PR; 46\%), 19 patients (41\%) with stable disease (SD), and 5 patients (11\%) with progressive disease, for an ORR of 48\% (95\% CI, 33\%-63\%). Additional long-term disease stabilization ( $\mathrm{SD} \geq 24$ weeks) occurred in eight patients, for an overall clinical benefit (CR + PR + SD $\geq 24$ weeks) of $68 \%$ (95\% CI, 51\%-81\%). Median TTP was 42 weeks (95\% CI, 26-72 weeks). Toxicity was generally mild. Grade 3 or 4 non-hematologic adverse effects included hypertension, transaminitis, and nausea/vomiting. Circulating endothelial cells (CECs) were correlated with ORR $(p<0.02)$, clinical benefit $(p<0.01)$, and improved PFS $(p<0.04)$. They concluded that treatment with metronomic capecitabine and cyclophosphamide in combination with bevacizumab was effective in MBC and was minimally toxic. The number of baseline CECs significantly correlated with response and outcome, supporting further studies on this surrogate marker for patient selection for antiangiogenic treatments.

\section{Safety and toxicity data with bevacizumab}

Bevacizumab appeared safe and manageable in patients with $\mathrm{MBC}$, with minimal additional toxicity seen when combined with other agents. However, the addition of bevacizumab increased the incidence of hypertension and proteinuria. Proteinuria is virtually always asymptomatic with similar rates reported in two phase 3 trials (only $1 \%$ reported grade 4 events). Mild proteinuria can be followed simply by periodic urinalysis, with concurrent monitoring for the development of hypertension. Higher levels of proteinuria should prompt 24-hour urine collection, and bevacizumab should be withheld for protein level $\geq 2 \mathrm{~g} / 24$ hours. The agent can be resumed with close observation when urine protein level falls below $2 \mathrm{~g} / 24$ hours. In E2100, grade 3 hypertension occurred in $15 \%$ of patients in the combination arm, compared with $2 \%$ in the single-agent paclitaxel arm. Similar rates were reported in the capecitabine trial $(17.9 \%$ vs $0.5 \%)$. There was no reported grade 4 hypertension in the capecitabine trial and $<1 \%$ reported in E2100. Hypertension can usually be managed with the addition of oral agents without the need for dose-reduction or discontinuation of bevacizumab; transient interruption of bevacizumab therapy may be required to allow adequate blood pressure control in rare patients. There is no consensus as to the ideal oral agent for the treatment 
of bevacizumab-induced hypertension. Some authors have suggested a preference for calcium-channel antagonists, while others favor angiotensin-converting-enzyme inhibitors, particularly in patients with coexisting proteinuria. Whether or not hypertension develops, close monitoring of blood pressure and urine protein levels is mandatory during bevacizumab treatment. Cumulative experience from multiple phase 3 trials, including those in patients with $\mathrm{MBC}$, confirms this experience. Additional severe adverse events have been reported with bevacizumab: hypertension, including hypertensive crisis; proteinuria, including nephritic syndrome; thrombosis (venous and arterial), including cerebral and myocardial infarction, transient ischemic attacks, and deep venous thrombosis; bleeding and hemorrage; impaired wound healing; gastrointestinal perforation; congestive heart failure (seen only in patients receiving anthracyclines and/or left chest-wall irradiation). These events are exceedingly rare, but do warrant consideration in selected patients for whom bevacizumab is planned (Midgley et al 2005). In breast cancer studies there has been some concern about the development of congestive heart failure (CHF), with an apparent, though non-significant, increase in events reported in the initial MBC with capecitabine study (Table 1). Current experience suggests that prior or concurrent anthracycline therapy or left-chest wall irradiation are risk factors for CHF during bevacizumab therapy. It is hoped that an ongoing trial in the adjuvant setting (E2104) will clarify the impact of bevacizumab on cardiac function (Miller et al 2007).

There has been some concern about risk for hemorrhage with the use of bevacizumab, particularly the potential for central nervous system (CNS) bleeds. While E2100 reported similar rates of minor mucosal bleeds for both treatment arms, the trial design intentionally excluded patients with CNS metastasis at the time of study accrual, given the unknown potential risk for hemorrhage at these sites. Because the safety of bevacizumab in patients with CNS metastasis remains unknown, the drug is best avoided in this setting for now. Clinicians should perform CNS imaging prior to therapy with bevacizumab to rule out occult metastasis (Genentech Inc.), and withhold the drug if detected. Venous and arterial thrombotic events also have been reported in other trials, including cerebral and myocardial infarction and pulmonary embolism. Clinicians should consider avoiding bevacizumab in patients with a history of prior thrombotic events and in patients receiving systemic anticoagulation, because the combination has not been studied in MBC. Patients with MBC on prophylactic aspirin (81-325 mg daily) have been safely treated with bevacizumab with no significant increased risk for bleeding.

Finally, a recent work supports an association between VEGF genotype and median OS as well as grade 3 or 4 hypertension when using bevacizumab in MBC. The VEGF-2578 AA genotype was associated with a superior median overall survival (OS) in the combination arm when compared with the alternative genotypes combined (HR, 0.58; 95\% CI, 0.36-0.93; $\mathrm{p}=0.023$ ). The VEGF-1154 A allele also demonstrated a superior median OS with an additive effect of each active allele in the combination arm but not the control arm (HR, 0.62; 95\% CI, 0.46-0.83; $\mathrm{p}=0.001)$. Two additional genotypes, VEGF-634 CC and

Table I Reported incidence of congestive heart failure with bevacizumab

\begin{tabular}{|c|c|c|}
\hline Study/Regimen & $\begin{array}{l}\text { Observed incidence of congestive } \\
\text { heart failure }\end{array}$ & Reference \\
\hline $\begin{array}{l}\text { Bevacizumab monotherapy in breast } \\
\text { cancer }\end{array}$ & $\begin{array}{l}2 / 75 \text { patients }(2.7 \%) \text { - both received } \\
\text { prior doxorubicin }\end{array}$ & Cobleigh et al 2003 \\
\hline $\begin{array}{l}\text { Capecitabine } \pm \text { bevacizumab in breast } \\
\text { cancer }\end{array}$ & $2(0.9 \%)$ vs 7 (3.1\%) & Miller et al 2006 \\
\hline $\begin{array}{l}\text { E2 } 100 \text { - bevacizumab + paclitaxel in breast } \\
\text { cancer }\end{array}$ & Preliminary data showing I/342 (0.3\%) & Miller et al 2007 \\
\hline $\begin{array}{l}\text { AML } \\
\text { (I-beta-d-arabinofuranosylcytosine, } \\
\text { mitoxantrone + bevacizumab) }\end{array}$ & $6 \%$ incidence of $\mathrm{CHF}$ & Karp et al 2004 \\
\hline $\begin{array}{l}\text { Neoadjuvant AT + bevacizumab } \\
\text { inflammatory breast }\end{array}$ & $2 / 21(9.5 \%)$ & Wedam et al 2006 \\
\hline $\begin{array}{l}\text { Doxorubicin + bevacizumab metastatic } \\
\text { sarcomas }\end{array}$ & $\begin{array}{l}\text { 2/I7 (II.8\%) CHF + 4/I7 (23.5\%) } \\
\text { decrease LVEF }\end{array}$ & D'Adamo et al 2005 \\
\hline
\end{tabular}

Abbreviations: AML, acute myeloid leukemia; AT, doxorubicin and docetaxel; CHF, congestive heart failure; LVEF, left-ventricular ejection fraction. 
VEGF-1498 TT, were associated with significantly less grade 3 or 4 hypertension in the combination arm when compared with the alternate genotypes combined $(p=0.005$ and $\mathrm{p}=0.022$, respectively) (Schneider et al 2008). This is a first report that needs to be further investigated to improve the use and safety of this drug.

\section{Ongoing trials in HER2-negative breast cancer patients}

Because antiangiogenic therapy has the potential to improve the clinical outcome in patients with $\mathrm{MBC}$, there is a need to optimize its use, particularly early in the course of metastatic disease.

The ongoing RiBBON studies are large, multicenter, randomized, placebo-controlled phase 3 trials that are evaluating the efficacy and safety of bevacizumab in combination with standard-of-care chemotherapy regimens in patients with previously untreated (RiBBON 1) or previously treated (RiBBON 2) MBC (O’Shaughnessy et al 2008). Because investigators are given the choice of which chemotherapy regimen to use, the $\mathrm{RiBBON}$ trials might provide data to support the use of bevacizumab in combination with various standard-of-care chemotherapy agents, including capecitabine. The capecitabine arm in this study is adequately powered for a statistically significant difference in capecitabine versus capecitabine/bevacizumab to be demonstrated. Another unique feature is that RiBBON 2 is a purely second-line study, in contrast with the AVF2119g trial, in which approximately $40 \%$ of patients had been treated with $\geq 2$ chemotherapy regimens for $\mathrm{MBC}$. Besides the use of bevacizumab in the metastatic setting, it has been hypothesized that the greater benefit could be obtained when used in earlier stages. The progression of breast cancer is accompanied by the production of a wide array of proangiogenic growth factors that promote and support tumor growth. When tumors are small they secrete VEGF, which acts as a paracrine factor to induce endothelial cell proliferation and blood vessel formation, mediating tumor progression. As the tumor develops further, additional factors are also secreted. This evidence suggests that the inhibition of VEGF may be more beneficial in earlier stages, as neoadjuvant or adjuvant treatment.

There are many trials, planned and ongoing, exploring bevacizumab in the neoadjuvant setting. An ongoing, phase 2 trial of bevacizumab in combination with docetaxel in the neoadjuvant setting (AVF2307s) (Cortés-Funes 2007) is being conducted in patients with locally unresectable breast cancer with or without metastasis. Patients receive bevacizumab $10 \mathrm{mg} / \mathrm{kg}$ every 2 weeks and docetaxel $35 \mathrm{mg} / \mathrm{m}^{2}$ weekly for the first 6 weeks of an 8-week cycle. After 2 cycles, patients with stable disease or response, undergo

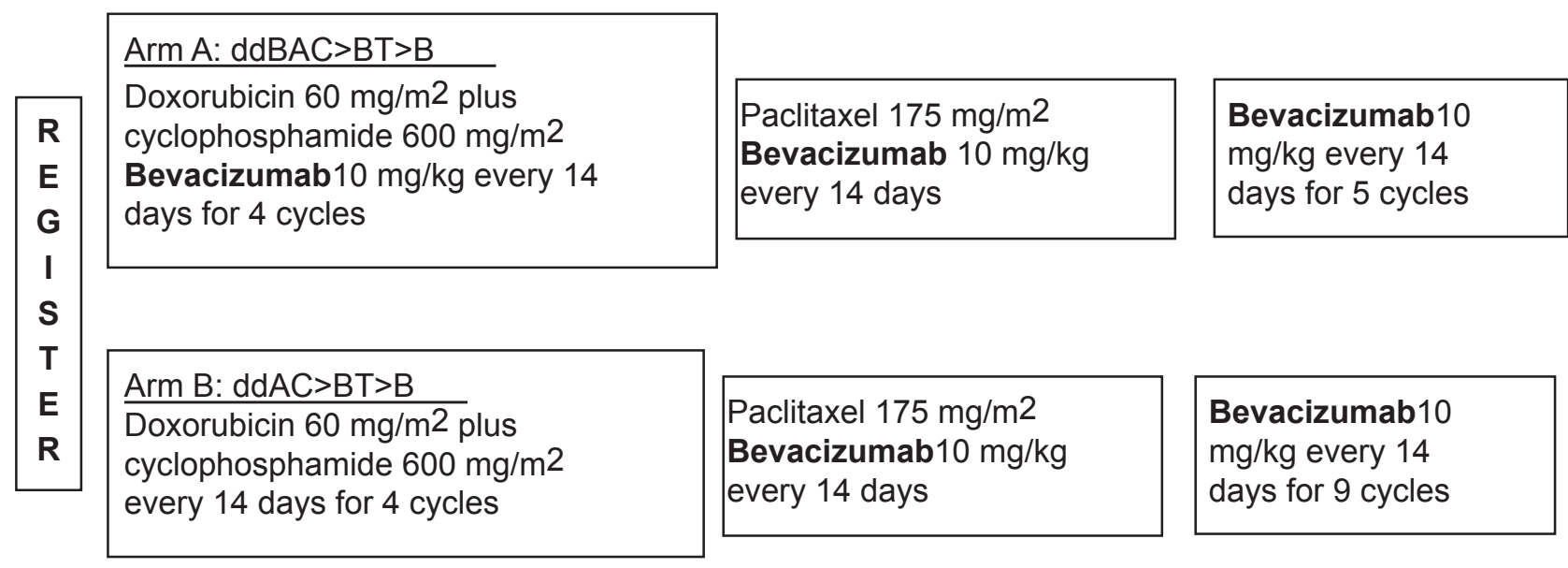

Key

Arm A: dd (dose dense) B (bevacizumab) A (doxorubicin) B (bevacizumab) T (paclitaxel) > B (bevacizumab)

Arm B: dd (dose dense) A (doxorubicin) C (cyclophosphamide) (paclitaxel) $>$ B (bevacizumab)

Figure I ECOG Study 2104. 
surgery and radiotherapy, followed by further chemotherapy (not containing bevacizumab). The study objectives are to evaluate the efficacy and safety of neoadjuvant bevacizumab with docetaxel in breast cancer patients.

TORI-B-02 (http://clinicaltrials.gov/ct2/show/NCT002 03372?term=TORI+B02\&rank=1) is a phase 2 neoadjuvant trial currently being conducted at the University of California, Los Angeles. A total of 90 patients are being enrolled into 4 different treatment arms, in order to compare 2 different bevacizumab doses with placebo: Arm 1: bevacizumab $7.5 \mathrm{mg} / \mathrm{kg}$, followed by 6 cycles of bevacizumab $7.5 \mathrm{mg} / \mathrm{kg}$ in combination with TAC (docetaxel, doxorubicin and cyclophosphamide) every 3 weeks; Arm 2: placebo $7.5 \mathrm{mg} / \mathrm{kg}$, followed by 6 cycles of placebo $7.5 \mathrm{mg} / \mathrm{kg}$ in combination with TAC every 3 weeks; Arm 3: bevacizumab $15 \mathrm{mg} / \mathrm{kg}$, followed by 6 cycles of bevacizumab $15 \mathrm{mg} / \mathrm{kg}$ in combination with TAC every 3 weeks; and Arm 4: placebo $15 \mathrm{mg} / \mathrm{kg}$, followed by 6 cycles of placebo $15 \mathrm{mg} / \mathrm{kg}$ in combination with TAC every 3 weeks. Then, 28 to 42 days after receiving chemotherapy, patients eligible for surgery will undergo resection. After either receiving surgery or completing chemotherapy, patients in Arms 1 and 3 will receive bevacizumab at their previous dose until disease progression. Patients in Arms 2 and 4 will not receive further therapy before disease progression. The primary objectives of the TORI-B-02 trial are to evaluate the safety and toxicity of bevacizumab given as preoperative therapy to patients with stage II/III breast cancer in combination with the TAC regimen. Analysis of changes in HIF-1 $\alpha$ levels will also be carried out, in order to compare tumor angiogenesis in patients given bevacizumab and placebo. Other trial objectives include investigation of the clinical benefit of adding bevacizumab to TAC in the neoadjuvant setting. Parameters to be evaluated are the clinical objective response rate (complete response plus partial response), pathologic complete response rate and rate of breast-conserving surgery. As wound-healing complications have previously been reported in bevacizumab clinical trials, the effect of bevacizumab on post-surgical wound-healing will also be investigated as well as the rate of cardiac heart failure.

Another ongoing study in the adjuvant setting is the BEATRICE study. This 2-arm open-label study will evaluate the efficacy and safety of the addition of bevacizumab to standard adjuvant therapy in patients with triple negative breast cancer. Patients will be randomized to receive either standard chemotherapy (anthracycline \pm taxane or taxane only), or standard chemotherapy given concurrently with 1 year of bevacizumab ( $5 \mathrm{mg} / \mathrm{kg} /$ week dosing equivalent iv). The anticipated time on study treatment is 3 to 12 months.
Target sample size is 2530 . The most successful use of antiangiogenic therapy has been predicted to be in the adjuvant treatment, and there is a clear biologic basis and rationale for exploring bevacizumab in this clinical setting. A large proportion of patients with breast cancer have been reported to have primary tumors that over express VEGF, and over expression of VEGF is associated with increased rates of relapse.

The trials planned with bevacizumab in the adjuvant setting will confirm the efficacy of adding an antiangiogenic therapy to standard combinations. The ECOG trial E2104 (Figure 1) (Miller et al 2007) is a 2-armed, non-randomized pilot study to evaluate the efficacy of bevacizumab as an adjuvant therapy in patients with HER2-negative, lymph node-positive early breast cancer. Accrual onto each arm of the trial was carried out sequentially in cohorts of 106 patients, beginning with arm A. Patients in arm A of the trial received 4 cycles of doxorubicin $\left(60 \mathrm{mg} / \mathrm{m}^{2}\right)$, cyclophosphamide $\left(600 \mathrm{mg} / \mathrm{m}^{2}\right)$ and bevacizumab $(10 \mathrm{mg} / \mathrm{kg})$ every 2 weeks, followed by 4 cycles of paclitaxel $\left(175 \mathrm{mg} / \mathrm{m}^{2}\right)$ and bevacizumab $(10 \mathrm{mg} / \mathrm{kg})$ every 2 weeks. Patients then received bevacizumab $(10 \mathrm{mg} / \mathrm{kg})$ alone for 18 2-week cycles. Patients in arm B of the trial received 4 cycles of doxorubicin $\left(60 \mathrm{mg} / \mathrm{m}^{2}\right)$ and cyclophosphamide $\left(600 \mathrm{mg} / \mathrm{m}^{2}\right)$ every 2 weeks, followed by 4 cycles of paclitaxel $\left(175 \mathrm{mg} / \mathrm{m}^{2}\right)$ and bevacizumab $(10 \mathrm{mg} / \mathrm{kg})$ every 2 weeks. This was followed by bevacizumab $(10 \mathrm{mg} / \mathrm{kg})$ alone for 22 -week cycles. Growth factor support with granulocyte colony stimulating factor was used during chemotherapy, but not required with single-agent bevacizumab. The primary endpoint is safety concerning the incidence of clinically apparent cardiac dysfunction. Preliminary safety data indicate that this combination is feasible, with cardiac toxicity within acceptable limits in both trial arms.

A planned 4950 patients will participate in the ECOG study E5103 (ecog.dfci.harvard.edu/general/videos/etpages/e5103. $\mathrm{html}$ ), a randomized, phase 3 trial to evaluate the efficacy and safety of bevacizumab as an adjuvant therapy in combination with chemotherapy. Eligible patients have ER-negative or high-risk estrogen-receptor positive, HER2-negative early breast cancer. Patients in arm A of the trial will receive 4 cycles of doxorubicin $\left(60 \mathrm{mg} / \mathrm{m}^{2}\right)$, cyclophosphamide $\left(600 \mathrm{mg} / \mathrm{m}^{2}\right)$ and placebo every 2 or 3 weeks (at the discretion of the investigator), followed by 12 cycles of weekly paclitaxel $\left(80 \mathrm{mg} / \mathrm{m}^{2}\right)$ plus placebo. Patients in arm B of the trial will receive 4 cycles of doxorubicin $\left(60 \mathrm{mg} / \mathrm{m}^{2}\right)$, cyclophosphamide $\left(600 \mathrm{mg} / \mathrm{m}^{2}\right)$ and bevacizumab $(15 \mathrm{mg} / \mathrm{kg})$ every 2 or 3 weeks, followed by 12 cycles of weekly paclitaxel $\left(80 \mathrm{mg} / \mathrm{m}^{2}\right)$ in combination with 4 cycles of bevacizumab $(15 \mathrm{mg} / \mathrm{kg})$ every 3 weeks. Patients in arm $\mathrm{C}$ of the trial will receive doxorubicin, 
cyclophosphamide and bevacizumab followed by weekly paclitaxel and bevacizumab as in arm B, with an additional ten 3-weekly cycles of bevacizumab $(15 \mathrm{mg} / \mathrm{kg})$. The primary trial endpoint is disease-free survival.

\section{Conclusion}

Angiogenesis has a clear and definite role in breast cancer progression and metastasis, making anti-VEGF therapies an attractive option for proceding with trials of efficacy. Bevacizumab is an effective adjunct in the treatment of MBC, particularly when combined with paclitaxel in the first-line setting. The drug is well tolerated, with minimal additional toxicity, but does require close monitoring of blood pressure and urine protein levels throughout treatment. Beyond the first-line setting, bevacizumab may be less beneficial, because tumors seemingly alter their angiogenesis pathways, relying less heavily on VEGF as they progress. Future research will seek to combine anti-VEGF agents in the neoadjuvant or adjuvant setting, where even more potential may exist for patients with breast cancer. The potential to use multiple targeted agents (eg, bevacizumab with trastuzumab) is also worthy of future consideration, and studies are currently exploring these strategies as well.

\section{Acknowledgments}

The autor would like to thank Dr V Saracino for manuscript editing and Dr U De Giorgi for careful English linguistic proofreading.

\section{Disclosures}

The has no conflicts of interest to disclose.

\section{References}

Avastin (bevacizumab) for intravenous use [package insert]. 2006. San Francisco, Calif. Genentech Inc., April.

Bocci G, Nicolaou KC, Kerbel RS. 2002. Protracted low-dose effects on human endothelial cell proliferation and survival in vitro reveal a selective antiangiogenic window for various chemotherapeutic drugs. Cancer Res, 62:6938-43.

Borgstrom P, Gold DP, Hillan KJ, et al. 1999. Importance of VEGF for breast cancer angiogenesis in vivo: implications from intravital microscopy of combination treatments with an anti-VEGF neutralizing monoclonal antibody and doxorubicin. Anticancer Res, 19:4203-14.

Burstein HJ, Parker LM, Savoie J, et al. 2002. Phase II trial of the anti-VEGF antibody bevacizumab in combination with vinorelbine for refractory advanced breast cancer. Breast Cancer Res Treat, 76(Suppl 1):S115.

Burstein HJ, Spigel D, Kindsvogel K, et al. 2005. Metronomic chemotherapy with and without bevacizumab for advanced breast cancer: A randomized phase II study. 28th Annual San Antonio Breast Cancer Symposium, San Antonio, TX, December 8-11, 2005 (abstr 4).

Cobleigh MA, Langmuir VK, Sledge GW, et al. 2003. A phase I/II doseescalation trial of bevacizumab in previously treated metastatic breast cancer. Semin Oncol, 30(5 Suppl 16):117-24.
Colleoni M, Rocca A, Sandri MT, et al. 2002. Low dose oral methotrexate and cyclophosphamide in metastatic breast cancer: Antitumor activity and correlation with vascular endothelial growth factor levels. Ann Oncol, 13:73-80.

Cortés-Funes H. 2007. Present and future roles of bevacizumab in breast cancer. Breast Cancer Res, 9(Suppl 1):S22.

D'Adamo DR, Anderson SE, Albritton K, et al. 2005. Phase II study of doxorubicin and bevacizumab for patients with metastatic soft-tissue sarcomas. J Clin Oncol, 23:7135-42.

Dellapasqua S, Bertolini F, Bagnardi V, et al. 2008. Metronomic cyclophosphamide and capecitabine combined with bevacizumab in advanced breast cancer. J Clin Oncol, 26: in press. ecog.dfci.harvard.edu/general/ videos/etpages/e5103.html.

Emmenegger U, Kerbel RS. 2007. Five years of clinical experience with metronomic chemotherapy: achievements and perspectives. Onkologie, 30:606-8.

European Medicines Agency. Avastin $25 \mathrm{mg} / \mathrm{mL}$ : summary of product characteristics [online]. URL: http:ww.emea.europa.eu/humandocs/ Humans/EPAR/avastin/avastin.htm.

Ferrara N, Davis-Smith T. 1997. The biology of vascular endothelial growth factor. Endocr Rev, 18:4-25.

Gasparini G, Longo R, Fanelli M, et al. 2005. Combination of antiangiogenic therapy with other anticancer therapies: results, challenges, and open questions. J Clin Oncol, 23:1295-311.

Genentech Inc. Avastin ${ }^{\mathrm{TM}}$ (bevacizumab): prescribing information [online]. URL: http://www.gene.com.

Gennari A, Conte P, Rosso R, et al. 2005. Survival of metastatic breast carcinoma patients over a 20 year period: A retrospective analysis based on individual patient data from six consecutive studies. Cancer, 104:1742-50.

Gordon MS, Margolin K, Talpaz M, et al. 2001. Phase I safety and pharmacokinetic study of recombinant human anti-vascular endothelial growth factor in patients with advanced cancer. J Clin Oncol, 19:843-50

Higgins B, Kolinsky K, Linn M, et al. 2007. Antitumor activity of capecitabine and bevacizumab combination in a human estrogen receptor-negative breast adenocarcinoma xenograft model. Anticancer Res, 27:2279-87.

http://clinicaltrials.gov/ct2/show/NCT00203372?term=TORI+B02\&rank=1. Jain RK. 2005. Normalization of tumor vasculature: an emerging concept in antiangiogenic therapy. Science, 307:58-62.

Kakeji Y, Teicher BA. 1997. Preclinical studies of the combination of angiogenic inhibitors with cytotoxic agents. Invest New Drugs, 15:39-48.

Karp JE, Gojo I, Pili R, et al. 2004. Targeting vascular endothelial growth factor for relapsed and refractory adult acute myelogenous leukemias: therapy with sequential 1-beta-d-arabinofuranosylcytosine, mitoxantrone, and bevacizumab. Clin Cancer Res, 10:3577-85.

Kerbel RS, Kamen BA. 2004. The anti-angiogenic basis of metronomic chemotherapy. Nat Rev Cancer, 4:423-36.

Kusaka M, Sudo K, Matsutani E, et al. 1994. Cytostatic inhibition of endothelial cell growth by the angiogenesis inhibitor TNP-470(AGM-1470). Br J Cancer, 69: 212-6.

Langmuir VK, Cobleigh MA, Herbst RS, et al. 2002. Successful long-term therapy with bevacizumab (Avastin ${ }^{\mathrm{TM}}$ ) in solid tumors. Proc Am Soc Clin Oncol, 1:9a. (Abstr 32).

Manley PW, Martiny-Baron G, Schlaeppi JM, et al. 2002. Therapies directed at vascular endothelial growth factor. Expert Opin Invest Drugs, 11:1715-36.

Margolin K, Gordon MS, Holmgren E, et al. 2001. Phase Ib trial of intravenous recombinant humanized monoclonal antibody to vascular endothelial growth factor in combination with chemotherapy in patients with advanced cancer: pharmacologic and long-term safety data. J Clin Oncol, 19:851-6.

Midgley R, Kerr D. 2005. Bevacizumab - current status and future directions. Ann Oncol, 16:999-1004. 
Miles D, Chan A, Romieu G, et al. 2008. Randomized, double blind, placebo controlled, phase III study of bevacizumab with docetaxel or docetaxel with placebo as first line therapy for patients with locally recurrent or metastatic breast cancer (mBC) AVADO. J Clin Oncol, (May 20 Suppl): abstr LBA1011.

Miller KD, Chap LI, Holmes FA, et al. 2005. Randomized phase III trial of capecitabine compared with bevacizumab plus capecitabine in patients with previously treated metastatic breast cancer. J Clin Oncol, 23:792-9.

Miller K, Gradishar W, Moish C, et al. 2006. Capecitabine plus bevacizumab in first line metastatic breast cancer: an interim safety and efficacy report of the first phase of Xeloda plus Avastin 1st line metastatic breast cancer trial. Breast Cancer Res Treat, 100(Suppl 1):S103 (Abstract 2068).

Miller KD, O’Neill A, Perez EA, et al. 2007. Phase II feasibility trial incorporating bevacizumab into dose dense doxorubicin and cyclophosphamide followed by paclitaxel in patients with lymph node positive breast cancer: a trial of the Eastern Cooperative Oncology Group (E2104). Breast Cancer Res Treat, 106(Suppl 1):S147 (Abstract 3063).

Miller KD, Wang, M, Gralow J, et al. 2007. Paclitaxel plus bevacizumab versus paclitaxel alone for metastatic breast cancer. $N$ Engl $J$ Med, 357.2666-76.

Muramaki M, Miyake H, Hara I, Kamidono S. 2005. Synergistic inhibition of tumor growth and metastasis by combined treatment with TNP-470 and docetaxel in a human prostate cancer PC-3 model. Int J Oncol, 26:623-8.
O'Shaughnessy JA, Brufsky AM. 2008. RiBBON 1 and RiBBON 2: phase III trials of bevacizumab with standard chemotherapy for metastatic breast cancer. Clin Breast Cancer, 8:370-3.

Polichemothearpy for Early Breast Cancer: an overview of the randomized trials. Early Breast Cancer Trialists' Collaborative Group. 1998. Lancet, 352:930-42.

Relf M, LeJeune S, Scott PA, et al. 1997. Expression of the angiogenic factors vascular endothelial cell growth factor, acidic and basic fibroblast growth factor, tumor growth factor beta-1, platelet-derived endothelial cell growth factor, placenta growth factor, and pleiotrophin in human primary breast cancer and its relation to angiogenesis. Cancer Res, 57:963-9.

Schneider BP, Wang M, Radovich M et al. 2008. Association of vascular endothelial growth factor and vascular endothelial growth factor receptor-2 genetic polymorphisms with outcome in a trial of paclitaxel compared with paclitaxel plus bevacizumab in advanced breast cancer: ECOG 2100. J Clin Oncol, 26:4672-8.

Slamon DJ, Romond EH, Perez EA. 2006. Advances in adjuvant therapy for breast cancer. Clin Adv Hematol Oncol, 4(Suppl 1):4-9.

Sweeney CJ, Miller KD, Sissons SE et al. 2001. The antiangiogenic property of docetaxel is synergistic with a recombinant humanized monoclonal antibody against vascular endothelial growth factor or 2-methoxyestradiol but antagonized by endothelial growth factors. Cancer Res, 61:3369-72.

Wedam SB, Low JA, Yang SX, et al. 2006. Antiangiogenic and antitumor effects of bevacizumab in patients with inflammatory and locally advanced breast cancer. J Clin Oncol, 24:769-77. 
